\title{
Recommendations for Managing Adults With Histiocytic Neoplasms: New NCCN Guidelines
}

\author{
Presented by Ronald S. Go, MD
}

\begin{abstract}
The histiocytoses, a group of clonal and reactive conditions, arise from monocytic macrophage or dendritic cell lineages. The current NCCN Clinical Practice Guidelines in Oncology (NCCN Guidelines) for Histiocytic Neoplasms reflect the most up-to-date, evidence-based data relating to the evaluation and management of this disease. Specifically, the guidelines focus on adult Langerhans cell histiocytosis, Erdheim-Chester disease, and RosaiDorfman disease. Because these disorders are rare, challenges have arisen regarding clinical suspicion, histologic diagnosis, treatment, and molecular subtyping. Future versions of the NCCN Guidelines will address the diagnosis and management of pediatric patients, as well as malignant histiocytosis.
\end{abstract}

J Natl Compr Canc Netw 2021;19(11.5):1355-1357 doi: $10.6004 /$ jnccn.2021.5103

\begin{abstract}
"Histiocytoses are rare diseases, and the annual incidences ... are not exactly known," revealed Ronald S. Go, MD, Associate Professor of Medicine, Mayo Clinic, Rochester, and panel chair of the NCCN Clinical Practice Guidelines in Oncology (NCCN Guidelines) for Histiocytic Neoplasms. Dr. Go presented these new guidelines at the NCCN 2021 Virtual Congress: Hematologic Malignancies.
\end{abstract}

\section{Overview of Histiocytic Disorders}

"In 2016, the Histiocyte Society put together a revised classification of histiocytoses, categorizing them into 5 subgroups," Dr. Go commented," "which included the Langerhans, cutaneous, Rosai-Dorfman, malignant, and hemophagocytic groups." The new NCCN Guidelines address the Langerhans and Rosai-Dorfman groups.

In terms of volume, the top 3 histiocytic neoplasms include Erdheim-Chester disease (ECD), Langerhans cell histiocytosis (LCH), and Rosai-Dorfman disease (RDD). According to Dr. Go, LCH and ECD are now known to be clonal in nature; however, RDD may be clonal or reactive. With the exception of LCH, these conditions had not been categorized as malignancies until recently-the annual incidence of LCH is approximately 2 to 3 cases per million people. "We do not know the incidences of RDD and ECD; however, based on various publications and estimates, the combined number of new diagnoses of $\mathrm{RDD}$ and ECD is likely less than a couple hundred cases per year," Dr. Go explained. “Therefore, for $\mathrm{LCH}, \mathrm{RDD}$, and ECD combined, the incidence is estimated to be approximately 1,000 new cases per year in the United States." ${ }^{2-4}$
These 3 conditions can be distinguished morphologically or immunohistochemically. According to Dr. Go, CD68, CD163, and S100 may be present in ECD, LCH, and $\mathrm{RDD}$; however, $\mathrm{S} 100$ is observed less frequently in patients with ECD. CD1A and CD207 are uniquely present in those with LCH. Factor XIIIa and Touton giant cells are commonly reported in ECD. Emperipolesis, which is the migration of inflammatory cells within the cytoplasm of histiocytes, is uniquely seen in patients with $\mathrm{RDD}$ (Table 1).5,6

Molecular alterations of BRAF V600E have been detected in approximately $50 \%$ to $60 \%$ of patients with ECD and LCH; other BRAF and MAPK pathway gene mutations have been found in this population (Table 1). Typically, in those with RDD, BRAF mutations do not occur; however, $M A P K$ pathway gene mutations are common. "Notably, [in] approximately $10 \%$ to $20 \%$ of cases, the mutations remain unknown," Dr. Go remarked. ${ }^{6}$

\section{Genesis of the NCCN Guidelines for Histiocytic Neoplasms}

"The idea to put together these NCCN Guidelines began in November 2018," Dr. Go commented. "IIn March 2021], the initial version of the guidelines was published on the NCCN website."

Because the Histiocyte Society had released guidelines regarding the management of pediatric histiocytic neoplasms, he explained that the initial focus of the NCCN Guidelines was on the adult diseases. Specifically, the NCCN Histiocytic Neoplasms Panel decided to focus on ECD, LCH, and RDD. Challenges have arisen regarding 


\begin{tabular}{|c|c|c|c|c|}
\hline & Tests & ECD & LCH & RDD \\
\hline CD68 & $\begin{array}{l}\text { Monocyte/Macrophage protein } \\
\text { Binds to tissue lectin/selectin }\end{array}$ & + & + & + \\
\hline CD163 & $\begin{array}{l}\text { Monocyte/Macrophage protein } \\
\text { Receptor for hgb-haptoglobin complex }\end{array}$ & + & + & + \\
\hline CD1a & $\begin{array}{l}\text { Related to } \mathrm{MHC} \text { proteins } \\
\text { Binds to b2-microglobulin }\end{array}$ & - & + & - \\
\hline CD207 & $\begin{array}{l}\text { Langerin; C-type lectin } \\
\text { Localized in Birbeck granules }\end{array}$ & - & + & - \\
\hline S100 & $\begin{array}{l}\text { Homodimeric polypeptides } \\
\text { Cell marker: neural crest, melanocyte }\end{array}$ & $+/-$ & + & + \\
\hline Factor XIIla & $\begin{array}{l}\text { Monocyte/Macrophage protein } \\
\text { AKA fibrin-stabilizing factor }\end{array}$ & + & - & - \\
\hline Touton giant cells & Macrophage-derived multinucleated giant cells with high lipid content & + & - & - \\
\hline Emperipolesis & Intact inflammatory cells within cytoplasm of histiocytes & - & - & + \\
\hline BRAF V600E & Proto-oncogene; cell division & $50 \%$ & $60 \%$ & $0 \%$ \\
\hline MAPK/ERK & Cell growth and proliferation & $40 \%$ & $30 \%$ & $40 \%$ \\
\hline
\end{tabular}

Abbreviations: ECD, Erdheim-Chester disease; LCH, Langerhans cell histiocytosis; MHC, major histocompatibility complex; RDD, Rosai-Dorfman disease.

the clinical suspicion, histologic diagnosis, treatment, and molecular subtyping of these disorders. "Treatment guidelines and follow-up are not based on a high level of evidence; most are based on expert recommendations," Dr. Go explained. "Certainly, clinical expertise is very helpful in the management of these patients."

\section{Erdheim-Chester Disease}

\section{NCCN Guidelines: Diagnosis}

According to Dr. Go, patients may present with one clinical manifestation of ECD and develop others over time. Frequently reported manifestations include skeletal, endocrine, central nervous system (CNS), cutaneous, retroperitoneal, arterial, and cardiac involvement. ${ }^{7}$ "[After a thorough review of medical history and complete physical examination], we recommend a PET/CT scan because many of the lesions appear fibrotic and are infiltrative in nature," Dr. Go commented. If PET/CT is unavailable, a whole-body CT scan, MRI of the brain, and bone scan may be performed as an alternative.

In regard to laboratory evaluation, a $\mathrm{CBC}$ count and chemistry panel are recommended in the NCCN Guidelines. Elevated levels of the C-reactive protein are frequently observed in patients with ECD; therefore, it may be used as a marker of disease activity and response. A panel should be ordered to examine anterior and posterior pituitary function. According to Dr. Go, tissue biopsy is essential; however, when biopsy is not possible, a presumptive diagnosis may be made based on the results of bone and PET scans.

"After a biopsy is performed, the typical morphology of ECD is the presence of foamy histiocytes and Touton giant cells," he remarked. "There will be a lot of fibrosis and inflammatory infiltrates." Immunohistochemistry, specifically BRAF V600E staining, should be performed to determine the subtype of histiocytosis; molecular studies may be performed to confirm these findings. A bone marrow biopsy may be considered if an unexplained CBC count abnormality is detected; however, in general, routine bone marrow biopsy is not recommended. ${ }^{8}$

\section{NCCN Guidelines: Treatment}

"As far as treatment of ECD is concerned, the first question is whether the patient is symptomatic," Dr. Go commented. "Also, is there any involvement of the critical organs, such as the brain, heart, or lungs?" If the patient is asymptomatic, and there is no impending organ dysfunction, the NCCN Guidelines suggest a "watch-andwait" approach. In those with symptomatic disease, systemic therapy may be considered.

Based on the guidelines, PET/CT scans should be performed every 3 to 6 months after the initiation of therapy. If there is brain involvement, Dr. Go noted that MRI may be a better imaging modality. Hormonal evaluations should be performed as needed.

"There are different options in terms of systemic therapy," he explained. "Knowing the type of mutation will help to determine which targeted agents to use." In general, patients with $B R A F$ V600E mutations will experience a response to vemurafenib; this is the only drug approved by the FDA for all histiocytoses. Those with MAPK pathway gene mutations may benefit from treatment with cobimetinib or other MEK inhibitors. Based on the guidelines, cladribine and pegylated interferon alpha-2a and alpha-2b may be administered irrespective of mutational status. ${ }^{8}$ 


\section{Langerhans Cell Histiocytosis}

\section{NCCN Guidelines: Diagnosis}

The most frequently reported presentations of LCH are lytic bone lesions and endocrine manifestations. Isolated pulmonary involvement is unique to $\mathrm{LCH}$ associated with smoking. Lymph node, cutaneous, and CNS involvement appears to be less common. ${ }^{7}$ According to Dr. Go, the clinical evaluation of this condition is very similar to that of ECD.

"For the tissue biopsy, the histiocytes are typically smaller compared [with] ECD, and they have grooved nuclei," he remarked. "The immunohistochemistry staining ... is quite unique to $\mathrm{LCH}$, with $\mathrm{CDla}$ and Langerin expression." Approximately half of patients seem to harbor $B R A F$ mutations; next-generation sequencing is recommended for confirmation. ${ }^{8}$

\section{NCCN Guidelines: Treatment}

For the treatment of LCH, Dr. Go explained that it is essential to determine the sites of involvement. Unifocal LCH, including isolated bone and skin disease, may be treated locally. In patients with advanced disease, systemic therapy should be used; the NCCN Guidelines recommend watch and wait as an alternative in asymptomatic cases.

Overall, the systemic therapy approach is similar in ECD and LCH. "The one thing distinct in LCH is that there are reports of the use of bisphosphonates for these lytic lesions, and [this therapy] can result in symptomatic improvement and sometimes partial and complete remissions," Dr. Go remarked. "Another thing that is unique to LCH is the pulmonary subtype, wherein the first management is smoking cessation; steroids may sometimes work, but systemic therapy used in the multisystem setting can also be used." The recommended follow-up approaches are also similar to those used in the management of ECD, according to Dr. Go. A PET/CT scan should be performed every 3 to 6 months during therapy.

"For systemic treatment, therapy is either targeted agents based on mutation or systemic chemotherapy irrespective of the mutation," he explained. "Cladribine has been studied extensively in LCH." The NCCN Guidelines also recommend cytarabine \pm methotrexate. ${ }^{8}$

"Of note, CNS lesions ... are very responsive to targeted agents," Dr. Go said. "Therefore, these agents may be the preferred treatment [in this patient population]."

\section{Rosai-Dorfman Disease}

\section{NCCN Guidelines: Diagnosis}

According to Dr. Go, lymph node manifestation of RDD is common in children; however, cutaneous involvement is more frequently reported in adults. Additional sites of involvement include the lymph nodes, bones, and retroorbital space. $^{7}$

Compared with ECD and $\mathrm{LCH}$, the evaluation and laboratory testing of RDD is similar. "One thing that is unique to RDD is that it can be associated with autoimmune disorders," Dr. Go remarked. "Therefore, autoimmune panels can be helpful in certain situations."

The typical morphology of RDD is the presence of histiocytes with round nuclei. Emperipolesis is typically observed in these lesions. ${ }^{8}$

\section{NCCN Guidelines: Treatment}

The NCCN Guidelines recommend the "watch-and-wait" approach in patients with asymptomatic RDD; this approach may also be used in those who do not have critical organ involvement. Patients with symptomatic RDD, especially those with cutaneous manifestations, may undergo surgical excision or resection. Multifocal diseases should be treated with systemic chemotherapy or targeted agents, according to Dr. Go. The drugs used in the treatment of RDD are similar to those administered in ECD and $\mathrm{LCH}^{8}$

Disclosures: Dr. Go has disclosed having no relevant financial relationships.

Correspondence: Ronald S. Go, MD, Mayo Clinic Cancer Center, 200 First Street SW, Rochester, MN 55905. Email: Go.Ronald@mayo.edu

\section{References}

1. Emile JF, Abla O, Fraitag S, et al. Revised classification of histiocytoses and neoplasms of the macrophage-dendritic cell lineages. Blood 2016; 127:2672-2681

2. Stålemark H, Laurencikas E, Karis J, et al. Incidence of Langerhans cell histiocytosis in children: a population-based study. Pediatr Blood Cancer 2008:51:76-81.

3. Goyal G, Shah MV, Hook CC, et al. Adult disseminated Langerhans cell histiocytosis: incidence, racial disparities and long-term outcomes. $\mathrm{Br} \mathrm{J}$ Haematol 2018;182:579-581.

4. Makras P, Stathi D, Yavropoulou M, et al. The annual incidence of Langerhans cell histiocytosis among adults living in Greece. Pediatr Blood Cancer 2020;67:e28422.
5. Diamond EL, Dagna L, Hyman DM, et al. Consensus guidelines for the diagnosis and clinical management of Erdheim-Chester disease. Blood 2014; 124:483-492.

6. Durham BH. Molecular characterization of the histiocytoses: neoplasia of dendritic cells and macrophages. Semin Cell Dev Biol 2019;86:62-76.

7. Goyal G, Young JR, Koster MJ, et al. The Mayo Clinic histiocytosis working group consensus statement for the diagnosis and evaluation of adult patients with histiocytic neoplasms: Erdheim-Chester disease, Langerhans cell histiocytosis, and Rosai-Dorfman disease. Mayo Clin Proc 2019;94:2054-2071.

8. Go RS, Jacobsen E, Baiocchi R, et al. NCCN Clinical Practice Guidelines in Oncology: Histiocytic Neoplasms. Version 2. 2021. Accessed September 21, 2021. To view the most recent version, visit NCCN.org 